\title{
EDITORIAL
}

\section{Winners of the 2016 JA Medals for excellence}

\author{
The Journal of Antibiotics (2017) 70, 1085-1086; doi:10.1038/ja.2017.133
}

$\mathrm{T}^{\mathrm{s}}$ he 2016 JA Medal for an original article is awarded for an excellent paper entitled 'Discovery of a capuramycin analog that kills nonreplicating Mycobacterium tuberculosis and its synergistic effects with translocase I inhibitors' by Shajila Siricilla, Katsuhiko Mitachi, Bajoie Wan, Scott G Franzblan and Michio Kurosu ${ }^{1}$ from University of Tennessee Health Science Center, Memphis and University of Illinois at Chicago, Chicago, IL, USA.

Current serious problems for tuberculosis (TB) control are longterm chemotherapy due to the nonreplicating (dormant) state of Mycobacterium tuberculosis (Mtb) and the occurrence of extensively drug resistant Mtb. The authors studied structure-activity relationships of capuramycin, a known inhibitor of translocase I (MurX/MreY or MurX for Mtb translocase I), involved in cell wall peptidoglycan biosynthesis. In this work, UT-01320, a synthetic $2^{\prime}$ methylated capuramycin analog, was found to kill both replicating and nonreplicating $\mathrm{Mtb}$ at low concentrations in in vitro assays. Surprisingly, UT-01320 does not inhibit MurX activity but potently blocks bacterial RNA polymerases, indicating that $O$-methylation at $2^{\prime}$ position alters its molecular target. Furthermore, UT-01320 displayed strong synergistic effects with MurX inhibitors such as capuromycin and the capuromycin analog SQ641, a preclinical drug. This work demonstrated a new type of bacterial RNA polymerase inhibitors targeting the dormant form of Mtb, leading to a promising TB chemotherapy. This paper also emphasizes the important concept that small change in antibiotic structure can result in significant impact on mode of action.

The 2016 JA Medal for reviews goes to an outstanding article entitled 'Glycopeptide antibiotic biosynthesis' by Gerard Wright and colleagues. $^{2}$ In this article, the authors report the current state of our knowledge of the biosynthesis of glycopeptide antibiotics (GPAs) such as vancomycin, the founding member of this class. GPAs are frequently used as a 'last resort' antibiotic treatment for many infections by Gram-positive bacteria, including methicillin-resistant Staphylococcus aureus. However, the emergence of vancomycinresistant enterococci (VRE) and its global spread as glycopeptide- resistant enterococci (GRE) have become a serious threat to the clinical community that has increasingly relied on GPAs to cure infectious disease, as a limited number of new antibiotics are in the pipeline. Exploration of GPA biosynthesis and elucidation of the resistance mechanism are key to achieving the goal of overcoming resistance with genetically and/or chemically modified GPAs.

The large and intricate chemical structures of GPAs consist of highly crosslinked and diversely modified core heptapeptides, synthesized by nonribosomal peptide synthetases (NRPS) and a variety of modification enzymes responsible for oxidative crosslinking, glycosylation, acylation, chlorination, sulfation, and methylation. This JA Medal winning group comprehensively describes detailed characterization of biosynthetic gene clusters revealed by whole-genome or meta-genome sequencing and the mechanism of self-resistance in producing microorganisms. Producers and other resistant organisms alter the target pentapeptide in Lipid II to terminate in D-Ala-DLactate rather than the canonical D-Ala-D-Ala, which reduces the affinity of GPAs by 1000-fold. Further understanding of both the biosynthesis of and resistance to GPAs will provide profound insights into strategies for the development of the next generation of GPAs to fight against GRE.

\section{CONFLICT OF INTEREST}

The author declare no conflict of interest.

Hiroshi Tomoda ${ }^{1}$ and Minoru Yoshida ${ }^{2}$

${ }^{1}$ Section Editor for New Anti-infective Substances, ${ }^{2}$ Review Editor,

The Journal of Antibiotics,

Japan Antibiotics Research Association,

Tokyo, Japan

1 Siricilla, S., Mitachi, K., Wan, B., Franzblau, S. G. \& Kurosu, M. Discovery of a capuramycin analog that kills nonreplicating Mycobacterium tuberculosis and its synergistic effects with translocase I inhibitors. J. Antibiot. 68, 271-278 (2015).

2 Yim, G., Thaker, M. N., Koteva, K. \& Wright, G. Glycopeptide antibiotic biosynthesis. J. Antibiot. 67, 31-41 (2014). 


\section{ABOUT THE WINNERS}

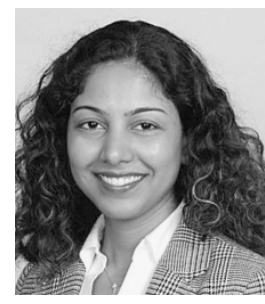

Dr Shajila Siricilla

Shajila Siricilla received her PhD in 2017 in Pharmaceutical Sciences from The University of Tennessee Health Science Center, Memphis, Tennessee under the supervision of Dr Michio Kurosu. During her PhD study, she worked on discovery of novel antibacterial molecules with novel molecular targets against problematic pathogens such as Mycobacterium tuberculosis and multidrug resistant nosocomial pathogens. She also worked on development of novel high-throughput amenable assays to screen compound libraries against polyprenyl-phosphate- $N$-acetylhexosamine-1-phosphate transferase enzyme superfamily and worked on validation of molecular targets of identified molecules that kill dormant Mycobacterium tuberculosis. After graduation, she joined Dr Jennifer Herington's group at Vanderbilt University Medical Center, Department of Pediatrics as a Postdoctoral Research Fellow. Dr Siricilla currently works on a project entailing a phenotypic high-throughput screen of large compound libraries to identify novel small molecules and molecular targets that regulate uterine myometrial contractions with high affinity and selectivity.

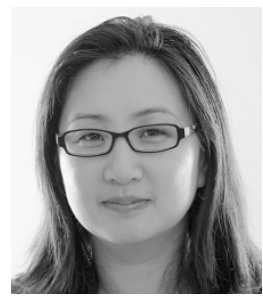

Dr Grace Yim

Grace Yim is a microbial geneticist and synthetic biologist specializing in antibiotics and their associated natural product biosynthesis. She obtained her B.Sc. in Biochemistry from the University of British Columbia and her Ph.D. from the University of British Columbia in the Department of Microbiology and Immunology under the supervision of Drs Julian Davies and George Spiegelman. During her time at UBC, she studied the molecular mechanisms by which sub-lethal concentrations of antimicrobials such as rifampicin, quinolones, macrolides, lincosamides and streptogramins affect bacterial transcription. After a short six-month postdoctoral fellowship with Dr Spiegelman, she began postdoctoral work in 2012 with Dr Gerry Wright at McMaster University. While at McMaster she has been utilizing genetic, biochemical and synthetic biology approaches to study antibiotic biosynthesis and to explore its application to drug discovery. She is studying glycopeptide biosynthesis and has engineered the bacteria Streptomyces coelicolor to heterologously express a glycopeptide biosynthetic gene cluster in various combinations with non-native modifying genes generating novel derivatives, expanding chemical diversity of this medically important class of antibiotic and illustrating important rules of modifying enzyme efficacy and regioselectivity necessary for combinatorial biosynthesis. Her fellowships include a NSERC doctoral fellowship, CIHR postdoctoral fellowship and a Michael G DeGroote postdoctoral fellowship. The resulting body of publications have over 1900 citations. 